\title{
ENTROPY AND APPROXIMATION NUMBERS OF EMBEDDINGS BETWEEN WEIGHTED BESOV SPACES
}

\author{
IWONA PIOTROWSKA \\ Mathematical Institute, Friedrich-Schiller-University \\ Ernst-Abbe-Platz 2, 07737 Jena, Germany \\ E-mail: iwona@minet.uni-jena.de
}

\begin{abstract}
The present paper is devoted to the study of the "quality" of the compactness of the trace operator. More precisely, we characterize the asymptotic behaviour of entropy numbers of the compact map

$$
\operatorname{tr}_{\Gamma}: B_{p_{1}, q}^{s}\left(\mathbb{R}^{n}, w_{\varkappa}^{\Gamma}\right) \rightarrow L_{p_{2}}(\Gamma)
$$

where $\Gamma$ is a $d$-set with $0<d<n$ and $w_{\varkappa}^{\Gamma}$ a weight of type $w_{\varkappa}^{\Gamma}(x) \sim \operatorname{dist}(x, \Gamma)^{\varkappa}$ near $\Gamma$ with $\varkappa>-(n-d)$. There are parallel results for approximation numbers.
\end{abstract}

1. Introduction. The aim of this paper is to study the compactness of the trace operator acting between a weighted Besov space and an approximation space on a fractal $d$-set $\Gamma$. More precisely, we use known results on entropy numbers in unweighted settings to investigate the behavior of entropy and approximation numbers of compact embeddings between weighted Besov spaces $B_{p q}^{s}\left(\mathbb{R}^{n}, w_{\varkappa}^{\Gamma}\right)$, where $w_{\varkappa}^{\Gamma}$ is a function that measures the distance of a given point $x \in \mathbb{R}^{n}$ to a certain fractal set $\Gamma, w_{\varkappa}^{\Gamma}(x)=\operatorname{dist}(x, \Gamma)^{\varkappa}$. In particular, we consider the trace operator from spaces $B_{p q}^{s}\left(\mathbb{R}^{n}, w_{\varkappa}^{\Gamma}\right)$ into Lebesgue spaces $L_{p}(\Gamma)$, where $\Gamma$ is a $d$-set. Furthermore, we generalize this concept to so-called $(d, \Psi)$-sets. We will study the weight function $v_{\varkappa}^{\Gamma}(x)=(\operatorname{dist}(x, \Gamma))^{\varkappa} \Psi(\operatorname{dist}(x, \Gamma))$, where $\Psi$ is an admissible function, see (2.7), and $\Gamma$ a corresponding $(d, \Psi)$-set. Moreover, we compute approximation numbers of the embeddings between function spaces of the above type.

The paper is organized as follows. In the next section we collect some notation and recall definitions and known results on Muckenhoupt weights. In particular, we define the weighted Besov spaces $B_{p q}^{s}\left(\mathbb{R}^{n}, w\right)$ with $w \in \mathcal{A}_{\infty}$. Moreover, we give the definition

2000 Mathematics Subject Classification: Primary 46E35; Secondary 42B35, 47B06.

Key words and phrases: entropy numbers, approximation numbers, weighted function spaces, Muckenhoupt weights, $d$-sets, $(d, \Psi)$-sets.

Research supported by Junior Research Team "Fractal Analysis".

The paper is in final form and no version of it will be published elsewhere. 
of Besov spaces of generalized smoothness and related $(d, \Psi)$-sets with an admissible function $\Psi$. The third section is devoted to traces of weighted spaces on $d$ - and $(d, \Psi)$ sets. We first give classical statements of the trace problem on $\mathbb{R}^{n-1}$. Subsequently we describe extensions of this problem to an arbitrary closed set $\Gamma \subset \mathbb{R}^{n}$ with $|\Gamma|=0$. We conclude this section by presenting recent results on traces of weighted Besov spaces on $d$ - and $(d, \Psi)$-sets [Pio] which are the main tool in prove our further results. The fourth section contains results on entropy numbers of the trace operator between weighted Besov spaces. More precisely, we investigate the asymptotic behavior of the entropy numbers of the compact embedding

$$
\text { id }: B_{p_{1} q_{1}}^{s_{1}}\left(\mathbb{R}^{n}, w_{\varkappa}^{\Gamma}\right) \rightarrow B_{p_{2} q_{2}}^{s_{2}}\left(\mathbb{R}^{n}, w_{\varkappa}^{\Gamma}\right) .
$$

Here $\Gamma$ denotes a $d$-set or $(d, \Psi)$-set. In the final section we give estimates of approximation numbers of a trace operator of weighted Besov spaces, e.g.

$$
e_{k}\left(\operatorname{tr}_{\Gamma}: B_{p p}^{s}\left(\mathbb{R}^{n}, w_{\varkappa}^{\Gamma}\right) \rightarrow L_{p}(\Gamma)\right) \sim k^{\frac{1}{d}\left(\frac{n+\varkappa}{p}-s\right)-\frac{1}{p}} \sim a_{k}\left(\operatorname{tr}_{\Gamma}: B_{p p}^{s}\left(\mathbb{R}^{n}, w_{\varkappa}^{\Gamma}\right) \rightarrow L_{p}(\Gamma)\right) .
$$

\section{Function spaces and weights}

2.1. Definitions. In this section we collect some notation that remains fixed throughout this paper. As usual, $\mathbb{R}^{n}$ denotes the $n$-dimensional real Euclidean space and the Euclidean scalar product of $x=\left(x_{1}, \ldots, x_{n}\right)$ and $y=\left(y_{1}, \ldots, y_{n}\right)$ is given by $x y=$ $x_{1} y_{1}+\cdots+x_{n} y_{n}$, as usual. Let $\mathbb{N}_{0}=\mathbb{N} \cup\{0\}$ stand for the non-negative integers.

We denote by $\mathcal{S}\left(\mathbb{R}^{n}\right)$ the Schwartz space of all complex-valued rapidly decreasing infinitely differentiable functions and by $\mathcal{S}^{\prime}\left(\mathbb{R}^{n}\right)$ its dual space of all tempered distributions on $\mathbb{R}^{n}$. As usual, the Fourier transform defined on $\mathcal{S}^{\prime}\left(\mathbb{R}^{n}\right)$ is given by

$$
\mathcal{F} f(\xi)=\widehat{f}(\xi)=(2 \pi)^{-n / 2} \int_{\mathbb{R}^{n}} f(x) e^{-i x \xi} \mathrm{d} x .
$$

Here $\mathrm{d} x$ denotes the $n$-dimensional Lebesgue measure. The Fourier transform is a one-toone mapping from $\mathcal{S}\left(\mathbb{R}^{n}\right)$ onto $\mathcal{S}\left(\mathbb{R}^{n}\right)$. As usual, $\mathcal{F}^{-1} f$ or $f^{\vee}$, stands for the inverse Fourier transform, given by the right-hand side of (2.1) with $i$ in place of $-i$. Let $\varphi \in \mathcal{S}\left(\mathbb{R}^{n}\right)$ with $\operatorname{supp} \varphi \subset\left\{y \in \mathbb{R}^{n}:|y|<2\right\}$ and $\varphi(x)=1$ if $|x| \leq 1$. Furthermore, let $\varphi_{0}=\varphi$ and for each $j \in \mathbb{N}$ we put

$$
\varphi_{j}(x)=\varphi\left(2^{-j} x\right)-\varphi\left(2^{-j+1} x\right)
$$

Then

$$
\sum_{j=0}^{\infty} \varphi_{j}(x)=1 \quad \text { for all } x \in \mathbb{R}^{n} .
$$

The system of functions $\left\{\varphi_{j}\right\}_{j=0}^{\infty}$ is called a smooth dyadic resolution of unity.

We will also need the following definition from fractal geometry.

Definition 2.1. Let $0<d<n$. A set $\Gamma \subset \mathbb{R}^{n}$ is called $d$-set if there exists a Borel measure $\mu$ in $\mathbb{R}^{n}$ such that $\operatorname{supp} \mu=\Gamma$ and there are constants $c_{1}, c_{2}>0$ such that for arbitrary $\gamma \in \Gamma$ and all $0<r<1$,

$$
c_{1} r^{d} \leq \mu(B(\gamma, r) \cap \Gamma) \leq c_{2} r^{d} .
$$


REMARK 2.2. Note that self-similar fractals are outstanding examples of $d$-sets. For instance, the Cantor set in $\mathbb{R}^{1}$ is a $d$-set for $d=\ln 2 / \ln 3$, and the Koch curve in $\mathbb{R}^{2}$ is a $d$-set for $d=\ln 4 / \ln 3$.

Let $\Gamma$ be a $d$-set and let $0<p \leq \infty$. Then $L_{p}(\Gamma)$ are the usual complex $L_{p}$-spaces with respect to the Borel measure $\mu$, quasi-normed by

$$
\left\|f \mid L_{p}(\Gamma)\right\|=\left(\int_{\Gamma}|f(\gamma)|^{p} \mu(\mathrm{d} \gamma)\right)^{1 / p}
$$

with usual modification for $p=\infty$.

2.2. Muckenhoupt weights and Besov spaces. We recall some known facts and definitions on $\mathcal{A}_{p}$ Muckenhoupt classes.

We say that $w$ belongs to the Muckenhoupt class $\mathcal{A}_{p}$ with $1<p<\infty$ if there exists a constant $0<A<\infty$ such that for all balls $B$ the following inequality holds

$$
\left(\frac{1}{|B|} \int_{B} w(x) \mathrm{d} x\right)^{1 / p} \cdot\left(\frac{1}{|B|} \int_{B} w(x)^{-p^{\prime} / p} \mathrm{~d} x\right)^{1 / p^{\prime}} \leq A,
$$

where $p^{\prime}$ is the dual exponent to $p$ given by $1 / p^{\prime}+1 / p=1$ and $|B|$ stands for the Lebesgue measure of the ball $B$.

Futhermore, let $M$ be the Hardy-Littlewood maximal operator given by

$$
M f(x)=\sup _{B(x, r) \in \mathcal{B}} \frac{1}{|B(x, r)|} \int_{B(x, r)}|f(y)| \mathrm{d} y, \quad x \in \mathbb{R}^{n},
$$

where $\mathcal{B}$ is the collection of all open balls $B(x, r)=\left\{y \in \mathbb{R}^{n}:|y-x|<r\right\}, r>0$. A weight $w$ belongs to the Muckenhoupt class $\mathcal{A}_{1}$ if there exists a constant $0<A<\infty$ such that the inequality

$$
M w(x) \leq A w(x)
$$

holds for almost all $x \in \mathbb{R}^{n}$.

We also consider the Muckenhoupt class $\mathcal{A}_{\infty}$ defined by

$$
\mathcal{A}_{\infty}=\bigcup_{p \geq 1} \mathcal{A}_{p}
$$

REMARK 2.3. The class of $\mathcal{A}_{p}$ weights was introduced by B. Muckenhoupt in [Muc72a] A systematic treatment of these classes of weights functions may be found, in particular, in the monographs [GR85], [ST89], and [Ste93, Chapter V].

The most famous example of a Muckenhoupt weight $w \in \mathcal{A}_{p}, 1<p<\infty$, is given by $w(x)=|x|^{\delta}$ with $-n<\delta<n(p-1)$. In the sequel, we are more interested in the following example.

ExAmPle 2.4. Let $\Gamma$ be a $d$-set with $0<d<n$ introduced in Definition 2.1 and let $\varkappa \in \mathbb{R}$. We study the weight $w_{\varkappa}^{\Gamma}(x), x \in \mathbb{R}^{n}$, given by

$$
w_{\varkappa}^{\Gamma}(x):= \begin{cases}\operatorname{dist}(x, \Gamma)^{\varkappa}, & \operatorname{dist}(x, \Gamma) \leq 1, \\ 1, & \text { otherwise. }\end{cases}
$$


Note that the weight function $w_{\varkappa}^{\Gamma}$ belongs to the Muckenhoupt class $\mathcal{A}_{p}$ for $1<p<\infty$ if, and only if, $-(n-d)<\varkappa<(n-d)(p-1)$. For the proof and more details we refer the reader to $[\mathrm{HP}]$.

Let $w \in \mathcal{A}_{\infty}$ according to (2.5). We define the weighted Lebesgue space $L_{p}\left(\mathbb{R}^{n}, w\right)$ with $0<p \leq \infty$ as the collection of all measurable functions such that

$$
\left\|f \mid L_{p}\left(\mathbb{R}^{n}, w\right)\right\|=\left(\int_{\mathbb{R}^{n}}|f(x)|^{p} w(x) \mathrm{d} x\right)^{1 / p}
$$

is finite. Note that corresponding space for $p=\infty$ coincides with the unweighted space $L_{\infty}\left(\mathbb{R}^{n}\right)$.

Definition 2.5. Let $0<p<\infty, 0<q \leq \infty, \quad s \in \mathbb{R}$ and let $\left\{\varphi_{j}\right\}_{j=0}^{\infty}$ be a smooth dyadic resolution of unity. Assume $w \in \mathcal{A}_{\infty}$. The weighted Besov space $B_{p q}^{s}\left(\mathbb{R}^{n}, w\right)$ is the set of all distributions $f \in \mathcal{S}^{\prime}\left(\mathbb{R}^{n}\right)$ such that

$$
\left\|f \mid B_{p q}^{s}\left(\mathbb{R}^{n}, w\right)\right\|=\left(\sum_{j=0}^{\infty} 2^{j s q}\left\|\mathcal{F}^{-1}\left(\varphi_{j} \mathcal{F} f\right) \mid L_{p}\left(\mathbb{R}^{n}, w\right)\right\|^{q}\right)^{1 / q}
$$

is finite. In the limiting case $q=\infty$ the usual modification is required.

REMARK 2.6. The above definition does not depend on the choice of the resolution of unity $\left\{\varphi_{j}\right\}_{j=0}^{\infty}$. The spaces $B_{p q}^{s}\left(\mathbb{R}^{n}, w\right)$ are quasi-Banach spaces (Banach spaces for $p, q \geq$ $1)$. Furthermore, it is clear that $\mathcal{F}^{-1}\left(\varphi_{j} \mathcal{F} f\right)$ is an entire analytic function on $\mathbb{R}^{n}$ for any $f \in \mathcal{S}^{\prime}\left(\mathbb{R}^{n}\right)$. We have, in particular, that $\mathcal{F}^{-1}\left(\varphi_{j} \mathcal{F} f\right)(x)$ make sense pointwise. One can also consider the weighted Triebel-Lizorkin spaces $F_{p q}^{s}\left(\mathbb{R}^{n}, w\right), 0<p<\infty, 0<q \leq \infty$, $s \in \mathbb{R}$, by interchanging the order of $\ell_{q^{-}}$and $L_{p}$-quasi-norms in (2.6), see [Bui82], [Bui84] and also $[\mathrm{HP}],[\mathrm{Pio06}]$ and references therein. Moreover, for the weight function $w \equiv 1$ we obtain classical (unweighted) versions of these spaces. The best references here are the monographs by H. Triebel [Tri83], [Tri92] and [Tri06]. A systematic treatment of the Besov spaces with weights from the class $\mathcal{A}_{\infty}$ is due to H. Q. Bui in [Bui82], [Bui84] with subsequent papers [BPT96] and [BPT97].

2.3. Besov spaces of generalized smoothness and $(d, \Psi)$-sets. Now, we recall definitions and results on spaces of generalized smoothness and related $(d, \Psi)$-sets that will be of importance in the next section.

Definition 2.7. A positive monotone function $\Psi$ on the interval $(0,1]$ is called admissible if

$$
\Psi\left(2^{-k}\right) \sim \Psi\left(2^{-2 k}\right), \quad k \in \mathbb{N}_{0} .
$$

EXAMPLE 2.8. Let $b \in \mathbb{R}$. Then

$$
\Psi_{b}(x)=(1+|\log (x)|)^{b}, \quad x \in(0,1],
$$

where the $\log$ is taken with respect to base 2 , is an admissible function according to the above definition. 
Definition 2.9. Let $\Gamma$ be a non-empty closed subset of $\mathbb{R}^{n}$.

(i) Let $0<d<n$ and let $\Psi$ be an admissible function according to Definition 2.7. Then $\Gamma$ is called a $(d, \Psi)$-set if there exist a Radon measure $\mu$ on $\mathbb{R}^{n}$ with $\operatorname{supp} \mu=\Gamma$ and two positive constants $c_{1}$ and $c_{2}$ such that

$$
c_{1} r^{d} \Psi(r) \leq \mu(B(\gamma, r)) \leq c_{2} r^{d} \Psi(r)
$$

for any ball $B(\gamma, r)$ in $\mathbb{R}^{n}$ centered at $\gamma \in \Gamma$ and of radius $r \in(0,1)$.

(ii) Let $\Psi$ be a decreasing admissible function according to Definition 2.7 with $\Psi(x) \rightarrow$ $\infty$, if $x \rightarrow 0$. Then $\Gamma$ is called an $(n, \Psi)$-set if there is a Radon measure $\mu$ in $\mathbb{R}^{n}$ with the above properties and $d=n$ in (2.8).

REMARK 2.10. Obviously, for $\Psi \equiv 1$ we obtain $d$-sets with $0<d<n$ as introduced in Definition 2.1. Let $0<d<n$ and let $\Psi$ be an admissible function, then for any couple $(d, \Psi)$ there exists a $(d, \Psi)$-set in $\mathbb{R}^{n}$, see [ET99, Proposition 2.8]. Furthermore any $(d, \Psi)$ set in $\mathbb{R}^{n}$ with $d<n$ satisfies the so-called ball condition, for more information see [Tri01, Proposition 22.6(iv)].

Let $\left\{\varphi_{j}\right\}_{j=0}^{\infty}$ be a smooth resolution of unity given by (2.2) and (2.3).

Definition 2.11. Let $0<p, q \leq \infty$ and $s \in \mathbb{R}$. Moreover, let $\Psi$ be an admissible function according to Definition 2.7. Then $B_{p q}^{s, \Psi}\left(\mathbb{R}^{n}\right)$ is the collection of all tempered distributions $f \in \mathcal{S}^{\prime}\left(\mathbb{R}^{n}\right)$ for which

$$
\left\|f \mid B_{p q}^{s, \Psi}\left(\mathbb{R}^{n}\right)\right\|=\left(\sum_{j=0}^{\infty} 2^{j s q} \Psi\left(2^{-j}\right)^{q}\left\|\left(\varphi_{j} \widehat{f}\right)^{\vee} \mid L_{p}\left(\mathbb{R}^{n}\right)\right\|^{q}\right)^{1 / q}
$$

(with the usual modification for $q=\infty$ ) is finite.

REMARK 2.12. The spaces $B_{p q}^{s, \Psi}\left(\mathbb{R}^{n}\right)$ were introduced by D. E. Edmunds and H. Triebel in [ET98]. For a complete treatment of these spaces we refer the reader to the work of S. D. Moura, [Mou01], see also [ET96], [Tri97] and [Tri01] for more details. One may also consider the Triebel-Lizorkin spaces of generalized smoothness $F_{p q}^{s, \Psi}\left(\mathbb{R}^{n}\right), 0<p<\infty$, $0<q \leq \infty, s \in \mathbb{R}$, by interchanging the order of $\ell_{q^{-}}$and $L_{p^{-}}$quasi-norms in (2.9). The spaces $B_{p q}^{s, \Psi}\left(\mathbb{R}^{n}\right)$ are quasi-Banach spaces (Banach spaces if $p \geq 1$ and $q \geq 1$ ). It is known that the space $B_{p q}^{s, \Psi}\left(\mathbb{R}^{n}\right)$ does not depend on the chosen smooth resolution of unity $\left\{\varphi_{j}\right\}_{j=0}^{\infty}$ (in the sense of equivalent quasi-norms). Taking $\Psi \equiv 1$ we obtain the classical Besov spaces $B_{p q}^{s}\left(\mathbb{R}^{n}\right)$.

We give an extension of Example 2.4 to $(d, \Psi)$-sets.

ExAmPle 2.13. Let $\Gamma$ be a $(d, \Psi)$-set, $0<d<n, \Psi$ an admissible function, $\varkappa \in \mathbb{R}$, and $v_{\varkappa}^{\Gamma}(x):= \begin{cases}(\operatorname{dist}(x, \Gamma))^{\varkappa} \Psi(\operatorname{dist}(x, \Gamma)), & \text { for } \operatorname{dist}(x, \Gamma) \leq 1, \\ \Psi(1), & \text { otherwise. }\end{cases}$

Analogously to the $d$-set case we obtain that $v_{\varkappa}^{\Gamma} \in \mathcal{A}_{\infty}$ if, and only if, $-(n-d)<\varkappa<$ $(n-d)(p-1)$, see [Pio06, Proposition 5.6]. 


\section{Traces on fractals of weighted Besov spaces}

3.1. Preliminaries. This section gives a brief survey of the results on trace problems of Besov spaces on fractals. Our choice of fractal sets are $d$ - and $(d, \Psi)$-sets. Let $x=$ $\left(x^{\prime}, x_{n}\right) \in \mathbb{R}^{n}$ with $x^{\prime} \in \mathbb{R}^{n-1}$. Recall that the trace of $f$ on $\mathbb{R}^{n-1}$ is the mapping

$$
\operatorname{tr}_{\mathbb{R}^{n-1}}: f(x) \mapsto f\left(x^{\prime}, 0\right) .
$$

In other words, $\operatorname{tr}_{\mathbb{R}^{n-1}}$ restricts functions on $\mathbb{R}^{n}$ to the hyperplane $H=\left\{x \in \mathbb{R}^{n}: x_{n}=\right.$ $0\}$. Given a function space $X \subset \mathcal{D}^{\prime}\left(\mathbb{R}^{n}\right)$, the trace problem consists in finding a space $Y \subset \mathcal{S}^{\prime}\left(\mathbb{R}^{n-1}\right)$ such that $\operatorname{tr}_{\mathbb{R}^{n-1}}$ is a bounded linear surjection from $X$ to $Y$. We refer to [Tri92, Section 4.4.1 and 4.4.2] for the classical trace problem. We shall explain the meaning of the trace (3.1) if we consider a suitable compact $d$-set instead of $\mathbb{R}^{n-1}$. We will interpret any function $f^{\Gamma} \in L_{p}(\Gamma), 1 \leq p<\infty$, as a tempered distribution $f \in \mathcal{S}^{\prime}\left(\mathbb{R}^{n}\right)$ given by

$$
f(\varphi)=\int_{\Gamma} f^{\Gamma}(\gamma)(\varphi \mid \Gamma)(\gamma) \mu(\mathrm{d} \gamma), \quad \varphi \in \mathcal{S}\left(\mathbb{R}^{n}\right)
$$

where the restriction $\varphi \mid \Gamma$ of $\varphi$ is understood pointwise and $\mu$ is a Radon measure on $\Gamma$. Let $\Gamma$ be a closed set in $\mathbb{R}^{n}$ with $|\Gamma|=0$. We assume that there exists a Radon measure $\mu$ on $\mathbb{R}^{n}$ with $\operatorname{supp} \mu=\Gamma$. The restriction $\operatorname{tr}_{\Gamma} \varphi=\varphi \mid \Gamma$ understood pointwise is well-defined for any $\varphi \in \mathcal{S}\left(\mathbb{R}^{n}\right)$. Moreover, let us suppose that for $s>0$ and $0<p, q<\infty$ there is a constant $c>0$ such that for all $\varphi \in \mathcal{S}\left(\mathbb{R}^{n}\right)$,

$$
\left\|\operatorname{tr}_{\Gamma} \varphi\left|L_{p}(\Gamma)\|\leq c\| \varphi\right| B_{p q}^{s}\left(\mathbb{R}^{n}, w_{\varkappa}^{\Gamma}\right)\right\| .
$$

Since the Schwartz class $\mathcal{S}\left(\mathbb{R}^{n}\right)$ is dense in $B_{p q}^{s}\left(\mathbb{R}^{n}, w_{\varkappa}^{\Gamma}\right)$, the inequality (3.2) may be extended by completion to all $f \in B_{p q}^{s}\left(\mathbb{R}^{n}, w_{\varkappa}^{\Gamma}\right)$. The resulting limit of $\operatorname{tr}_{\Gamma} \varphi$ will be denoted by $\operatorname{tr}_{\Gamma} f$. Note that it is independent of the approximation of $f \in B_{p q}^{s}\left(\mathbb{R}^{n}, w_{\varkappa}^{\Gamma}\right)$ by $\mathcal{S}\left(\mathbb{R}^{n}\right)$-functions due to $(3.2)$.

3.2. Traces of weighted Besov spaces on d-sets. In this subsection we present recent results for the trace problem of weighted Besov spaces. For proofs and more details from this and the next subsection we refer the reader to [Pio], [Pio06].

Theorem 3.1. Let $0<d<n, \varkappa>-(n-d), 0<p<\infty, 0<q \leq \min (1, p)$ and let $\Gamma$ be a d-set. Then we have

$$
\operatorname{tr}_{\Gamma} B_{p q}^{\frac{\varkappa}{p}+\frac{n-d}{p}}\left(\mathbb{R}^{n}, w_{\varkappa}^{\Gamma}\right)=L_{p}(\Gamma)
$$

in the sense that $\operatorname{tr}_{\Gamma} f \in L_{p}(\Gamma)$ for any $f \in B_{p q}^{\frac{\varkappa}{p}+\frac{n-d}{p}}\left(\mathbb{R}^{n}, w_{\varkappa}^{\Gamma}\right)$ and any $f^{\Gamma} \in L_{p}(\Gamma)$ is a trace of a suitable $g \in B_{p q}^{\frac{\varkappa}{p}+\frac{n-d}{p}}\left(\mathbb{R}^{n}, w_{\varkappa}^{\Gamma}\right)$ on $\Gamma$ and

$$
\left\|f^{\Gamma}\left|L_{p}(\Gamma)\|\sim \inf \| g\right| B_{p q}^{\frac{\varkappa}{p}+\frac{n-d}{p}}\left(\mathbb{R}^{n}, w_{\varkappa}^{\Gamma}\right)\right\|,
$$

where the infimum is taken over all $g \in B_{p q}^{\frac{\varkappa}{p}+\frac{n-d}{p}}\left(\mathbb{R}^{n}, w_{\varkappa}^{\Gamma}\right)$ such that $\operatorname{tr}_{\Gamma} g=f^{\Gamma}$.

Motivated by the quasi-norm (3.4) we introduce the following trace spaces. 
Definition 3.2. Let $\Gamma$ be a $d$-set in $\mathbb{R}^{n}$ according to Definition 2.1 with $0<d<n$. Let $s>0,0<p \leq \infty$, and $0<q \leq \infty$. Let us define

$$
\mathbb{B}_{p q}^{s}(\Gamma)=\operatorname{tr}_{\Gamma} B_{p q}^{s+\frac{n-d}{p}}\left(\mathbb{R}^{n}\right) .
$$

We equip this space with the quasi-norm

$$
\left\|f\left|\mathbb{B}_{p q}^{s}(\Gamma)\|=\inf \| g\right| B_{p q}^{s+\frac{n-d}{p}}\left(\mathbb{R}^{n}\right)\right\|,
$$

where the infimum ranges over all $g \in B_{p q}^{s+\frac{n-d}{p}}\left(\mathbb{R}^{n}\right)$ with $\operatorname{tr}_{\Gamma} g=f$.

TheOREM 3.3. Let $0<d<n, s>0,0<p<\infty, 0<q \leq \infty$ and $-(n-d)<\varkappa<$ $s p-(n-d)$. Then

$$
\operatorname{tr}_{\Gamma} B_{p q}^{s}\left(\mathbb{R}^{n}, w_{\varkappa}^{\Gamma}\right)=\mathbb{B}_{p q}^{s-\frac{n-d}{p}-\frac{\varkappa}{p}}(\Gamma) .
$$

3.3. Traces of weighted Besov spaces on $(d, \Psi)$-sets. We begin with the counterpart of Definition 3.2 for the trace spaces with respect to the Besov spaces of generalized smoothness.

Definition 3.4. Let $0<p, q \leq \infty, s>0, \Psi$ be an admissible function and let $\Gamma$ be a $(d, \Psi)$-set in $\mathbb{R}^{n}$ with $0<d<n$. We define

$$
\mathbb{B}_{p q}^{s}(\Gamma):=\operatorname{tr}_{\Gamma} B_{p q}^{s+\frac{n-d}{p}, \Psi^{1 / p}}\left(\mathbb{R}^{n}\right) .
$$

We equip this space with the quasi-norm

$$
\left\|f\left|\mathbb{B}_{p q}^{s}(\Gamma)\|=\inf \| g\right| B_{p q}^{s+\frac{n-d}{p}, \Psi^{1 / p}}\left(\mathbb{R}^{n}\right)\right\|,
$$

where the infimum is taken over all $g \in B_{p q}^{s+\frac{n-d}{p}, \Psi^{1 / p}}\left(\mathbb{R}^{n}\right)$ with $\operatorname{tr}_{\Gamma} g=f$.

Note that for $\Psi \equiv 1, \Gamma$ is a $d$-set according to Definition 2.1, and then the above definition covers Definition 3.2. Let $v_{\varkappa}^{\Gamma}$ be the Muckenhoupt weight introduced in Example 2.13. We have the following generalization of Theorem 3.3.

TheOrem 3.5. Let $0<d<n, s \in \mathbb{R},-(n-d)<\varkappa, 0<p<\infty, 0<q \leq \infty, \Psi$ be an admissible function and let $\Gamma$ be $a(d, \Psi)$-set according to Definition 2.9(i). Then

$$
\operatorname{tr}_{\Gamma} B_{p q}^{s}\left(\mathbb{R}^{n}, v_{\varkappa}^{\Gamma}\right)=\operatorname{tr}_{\Gamma} B_{p q}^{s-\frac{\varkappa}{p}, \Psi^{1 / p}}\left(\mathbb{R}^{n}\right),
$$

whenever these spaces exist. Moreover, when $-(n-d)<\varkappa<s p-(n-d)$, then

$$
\operatorname{tr}_{\Gamma} B_{p q}^{s}\left(\mathbb{R}^{n}, v_{\varkappa}^{\Gamma}\right)=\mathbb{B}_{p q}^{s-\frac{\varkappa}{p}-\frac{n-d}{p}}(\Gamma) .
$$

\section{Entropy numbers of embeddings between weighted Besov spaces}

4.1. Definitions. Let $X$ and $Y$ be quasi-Banach spaces and let $T: X \rightarrow Y$ be a bounded linear operator. Let

$$
U_{X}:=\{x \in X:\|x \mid X\| \leq 1\}
$$

be the unit ball in the quasi-Banach space $X$. An operator $T$ is called compact if for any given $\varepsilon>0$ we can cover the image of the unit ball $U_{X}$ with finitely many balls in $Y$ of radius $\varepsilon$. 
Definition 4.1. Let $X, Y$ be quasi-Banach spaces and let $T \in L(X, Y)$. Then for all $k \in \mathbb{N}$, the kth dyadic entropy number $e_{k}(T)$ of $T$ is defined by

$$
e_{k}(T)=\inf \left\{\varepsilon>0: T\left(U_{X}\right) \subset \bigcup_{j=1}^{2^{k-1}}\left(y_{j}+\varepsilon U_{Y}\right) \text { for some } y_{1}, \ldots, y_{2^{k-1}} \in Y\right\},
$$

where $U_{X}$ and $U_{Y}$ denote the unit balls in $X$ and $Y$, respectively.

These numbers have various elementary properties partly recalled in the following lemma.

Lemma 4.2. Let $X, Y$ and $Z$ be quasi-Banach spaces, let $S, T \in L(X, Y)$ and $R \in$ $L(Y, Z)$.

(i) (Monotonicity): $\|T\| \geq e_{1}(T) \geq e_{2}(T) \geq \cdots \geq 0$. Moreover $\|T\|=e_{1}(T)$, provided that $Y$ is a Banach space.

(ii) (Additivity): If $Y$ is a $p$-Banach space $(0<p \leq 1)$, then for all $j, k \in \mathbb{N}$

$$
e_{j+k-1}^{p}(S+T) \leq e_{j}^{p}(S)+e_{k}^{p}(T) .
$$

(iii) (Multiplicativity): For all $j, k \in \mathbb{N}$

$$
e_{j+k-1}(R T) \leq e_{j}(R) e_{k}(T) .
$$

(iv) (Compactness): $T$ is compact if, and only if, $\lim _{k \rightarrow \infty} e_{k}(T)=0$.

Proofs of the above properties may be found for instance in [ET96, Lemma 1.3.1/1]. For more information, we recommend the monographs [ET96] and [CS90].

REMARK 4.3. Let us briefly discuss the connection between eigenvalues of a compact linear map and its entropy numbers, though applications of that kind are out of the scope of this paper. Let $T: X \rightarrow X$ be a compact linear operator in a quasi-Banach space $X$ and let $\left(\lambda_{n}(T)\right)$ be the sequence of all nonzero eigenvalues of $T$, repeated according to algebraic multiplicity and ordered so that $\left|\lambda_{1}(T)\right| \geq\left|\lambda_{2}(T)\right| \geq \ldots \geq 0$. Then Carl's inequality states

$$
\left|\lambda_{n}(T)\right| \leq \sqrt{2} e_{n}(T), \quad n \in \mathbb{N} .
$$

General references here are again [ET96] and [CS90]. Based on this inequality, and having in mind application to spectral theory of certain pseudo-differential operators, there was initiated a program to investigate the behavior of the entropy numbers in the context of weighted function spaces of Besov and Triebel-Lizorkin type, see [ET96] and [HT94a], [HT94b]. For a recent account we refer to the series of papers by T. Kühn et al. [KLSS06a], [KLSS06b], [KLSS].

4.2. Results and proofs. Let us first recall a result for entropy numbers in the corresponding unweighted situation, see [Tri97, Theorem 20.6].

THEOREM 4.4. Let $\Gamma$ be a compact $d$-set in $\mathbb{R}^{n}$ with $0<d<n$ according to Definition 2.1. Let $\mathbb{B}_{p q}^{s}(\Gamma)$ be the spaces introduced in Definition 3.2, notationally complemented by $\mathbb{B}_{p q}^{0}(\Gamma)=L_{p}(\Gamma)$ for any $0<p \leq \infty$ and $0<q \leq \infty$. Let

$$
0 \leq s_{2}<s_{1}<\infty, 0<p_{1}, p_{2} \leq \infty, 0<q_{1}, q_{2} \leq \infty,
$$


and

$$
s_{1}-s_{2}-d\left(\frac{1}{p_{1}}-\frac{1}{p_{2}}\right)_{+}>0 .
$$

Then the embedding

$$
\text { id }: \mathbb{B}_{p_{1} q_{1}}^{s_{1}}(\Gamma) \rightarrow \mathbb{B}_{p_{2} q_{2}}^{s_{2}}(\Gamma)
$$

is compact and the related entropy numbers satisfy

$$
e_{k}(\mathrm{id}) \sim k^{-\frac{s_{1}-s_{2}}{d}}, \quad k \in \mathbb{N} .
$$

REMARK 4.5. Recall that equivalence $\sim$ in (4.2) means that there exist two positive numbers $c_{1}$ and $c_{2}$ such that for all $k \in \mathbb{N}$,

$$
c_{1} k^{-\frac{s_{1}-s_{2}}{d}} \leq e_{k}(\mathrm{id}) \leq c_{2} k^{-\frac{s_{1}-s_{2}}{d}} .
$$

Assume that $0<q \leq \infty$ and

$$
s-d\left(\frac{1}{p_{1}}-\frac{1}{p_{2}}\right)_{+}>0 .
$$

Then (4.2) with $s_{1}=s$ and $s_{2}=0$ can be rewritten in the form

$$
e_{k}\left(\operatorname{tr}_{\Gamma}: B_{p_{1} q}^{s+\frac{n-d}{p_{1}}}\left(\mathbb{R}^{n}\right) \rightarrow L_{p_{2}}(\Gamma)\right) \sim k^{-\frac{s}{d}}, \quad k \in \mathbb{N}
$$

For more details, see [Tri97, Chapter IV, p.172].

We can now present results on entropy numbers for weighted Besov spaces.

THEOREM 4.6. Let $\Gamma$ be a d-set in $\mathbb{R}^{n}$ with $0<d<n$ according to Definition 2.1. Let $\frac{n-d+\varkappa}{p_{2}} \leq s_{2}<s_{1}<\infty, 0<p_{1}, p_{2}<\infty, 0<q_{1}, q_{2} \leq \infty$, and

$$
-(n-d)<\varkappa<\min \left(s_{1} p_{1}, s_{2} p_{2}\right)-(n-d) \text {. }
$$

Let

$$
s_{1}-s_{2}>(\varkappa+n-d)\left(\frac{1}{p_{1}}-\frac{1}{p_{2}}\right)+d\left(\frac{1}{p_{1}}-\frac{1}{p_{2}}\right)_{+} .
$$

Then for the weight $w_{\varkappa}^{\Gamma}$ introduced in Example 2.4 the embedding

$$
\text { id : } \operatorname{tr}_{\Gamma} B_{p_{1} q_{1}}^{s_{1}}\left(\mathbb{R}^{n}, w_{\varkappa}^{\Gamma}\right) \longrightarrow \operatorname{tr}_{\Gamma} B_{p_{2} q_{2}}^{s_{2}}\left(\mathbb{R}^{n}, w_{\varkappa}^{\Gamma}\right)
$$

is compact for the related entropy numbers satisfy

$$
e_{k}(\mathrm{id}) \sim k^{-\frac{s_{1}-s_{2}}{d}+\left(\frac{\varkappa+n-d}{d}\right)\left(\frac{1}{p_{1}}-\frac{1}{p_{2}}\right)}, \quad k \in \mathbb{N} .
$$

Proof. The proof is a simple consequence of Theorem 3.3 and Theorem 4.4. We have

$$
\begin{aligned}
e_{k}\left(\mathrm{id}: \operatorname{tr}_{\Gamma} B_{p_{1} q_{1}}^{s_{1}}\left(\mathbb{R}^{n}, w_{\varkappa}^{\Gamma}\right) \longrightarrow\right. & \left.\operatorname{tr}_{\Gamma} B_{p_{2} q_{2}}^{s_{2}}\left(\mathbb{R}^{n}, w_{\varkappa}^{\Gamma}\right)\right) \\
& e_{k}\left(\mathrm{id}: \mathbb{B}_{p_{1} q_{1}}^{s_{1}-\frac{\varkappa}{p_{1}}-\frac{n-d}{p_{1}}}(\Gamma) \longrightarrow \mathbb{B}_{p_{2} q_{2}}^{s_{2}-\frac{\varkappa}{p_{2}}-\frac{n-d}{p_{2}}}(\Gamma)\right) .
\end{aligned}
$$

By virtue of $(4.2)$ with $\bar{s}_{1}-\bar{s}_{2}-d\left(\frac{1}{p_{1}}-\frac{1}{p_{2}}\right)_{+}>0$ we obtain

$$
e_{k}\left(\mathrm{id}: \mathbb{B}_{p_{1} q_{1}}^{s_{1}-\frac{\varkappa}{p_{1}}-\frac{n-d}{p_{1}}}(\Gamma) \longrightarrow \mathbb{B}_{p_{2} q_{2}}^{s_{2}-\frac{\varkappa}{p_{2}}-\frac{n-d}{p_{2}}}(\Gamma)\right) \sim k^{-\frac{\bar{s}_{1}-\bar{s}_{2}}{d}}, k \in \mathbb{N}
$$

where 


$$
\bar{s}_{i}=s_{i}-\frac{\varkappa}{p_{i}}-\frac{n-d}{p_{i}} \text { for } i=1,2 .
$$

One immediately checks the compatibility of (4.4) and (4.5). This finishes the proof.

REMARK 4.7. Let $s=\delta+\frac{n-d}{p_{1}}+\frac{\varkappa}{p_{1}}$. From Theorem 3.3 we conclude that

$$
\operatorname{tr}_{\Gamma} B_{p_{1} q}^{s}\left(\mathbb{R}^{n}, w_{\varkappa}^{\Gamma}\right)=\mathbb{B}_{p_{1} q}^{\delta}(\Gamma), \quad \delta>0
$$

Furthermore, by Definition 13 we get

$$
\mathbb{B}_{p_{1} q}^{\delta}(\Gamma)=\operatorname{tr}_{\Gamma} B_{p_{1} q}^{\delta+\frac{n-d}{p_{1}}}\left(\mathbb{R}^{n}\right)
$$

Comparing this with (4.3) and the above theorem we obtain the following result.

Proposition 4.8. Let $\Gamma$ be a d-set in $\mathbb{R}^{n}$ with $0<d<n$ according to Definition 2.1. Let $0<p_{1}, p_{2}<\infty, 0<q \leq \infty,-(n-d)<\varkappa<s p_{1}-(n-d)$, and let $w_{\varkappa}^{\Gamma}$ be a weight function given by Example 2.4. Moreover, let

$$
s-\frac{1}{p_{1}}(\varkappa+n-d)-d\left(\frac{1}{p_{1}}-\frac{1}{p_{2}}\right)_{+}>0 .
$$

The trace operator $\operatorname{tr}_{\Gamma}$ of $B_{p_{1} q}^{s}\left(\mathbb{R}^{n}, w_{\varkappa}^{\Gamma}\right)$ into $L_{p_{2}}(\Gamma)$ is compact and the related entropy numbers satisfy

$$
e_{k}\left(\operatorname{tr}_{\Gamma}: B_{p_{1} q}^{s}\left(\mathbb{R}^{n}, w_{\varkappa}^{\Gamma}\right) \rightarrow L_{p_{2}}(\Gamma)\right) \sim k^{\frac{1}{d}\left(\frac{n+\varkappa}{p_{1}}-s\right)-\frac{1}{p_{1}}}
$$

One can extend this result to the $(d, \Psi)$-sets, where $\Psi$ is an admissible function according to Definition 2.7. In [ET99, Theorem 2.24] there is a generalization restricted to $1<p_{1}, p_{2} \leq \infty$ and the target spaces $L_{p}$. The case $0<p \leq 1$ has been considered by S. D. Moura in [Mou01,Theorem 3.3.2]. She dealt with target spaces of Besov type. Let $\Psi$ be an admissible function according to Definition 2.7 and let $\Gamma$ be a $(d, \Psi)$-set according to Definition 2.9. Let now $\mathbb{B}_{p q}^{s}(\Gamma)$ be the trace spaces introduced in Definition 3.4. By assumption we have $0<p_{1}, p_{2} \leq \infty, 0<q_{1}, q_{2} \leq \infty$, and $s_{1} \geq s_{2} \geq 0$ with

$$
s_{1}-s_{2}-d\left(\frac{1}{p_{1}}-\frac{1}{p_{2}}\right)_{+}>0
$$

such that the embedding

$$
\text { id }: \mathbb{B}_{p_{1} q_{1}}^{s_{1}}(\Gamma) \rightarrow \mathbb{B}_{p_{2} q_{2}}^{s_{2}}(\Gamma)
$$

is compact. Furthermore, the related entropy numbers satisfy

$$
e_{k}(\mathrm{id}) \sim\left[k \Psi\left(k^{-1}\right)\right]^{-\frac{s_{1}-s_{2}}{d}}, \quad k \in \mathbb{N} .
$$

(Note that we take $a_{1}=a_{2}=0$ in the original version of Theorem 3.3.2 in [Mou01], such that Definition 2.2.7 in [Mou01] of $\mathbb{B}$-spaces covers Definition 3.4.) The best general reference here is [Mou01, Chapter 3] and also [ET98] and [ET99].

We can now give an extension of Theorem 4.6 to $(d, \Psi)$-sets.

Proposition 4.9. Let $\Psi$ be an admissible function, and let $\Gamma$ be a $(d, \Psi)$-set according to Definition 2.9. Let $0<p_{1}, p_{2}<\infty, 0<q_{1}, q_{2} \leq \infty$, and

$$
-(n-d)<\varkappa<\min \left(s_{1} p_{1}, s_{2} p_{2}\right)-(n-d) .
$$


Moreover let $s_{1}, s_{2}$ be as in (4.5) with $s_{2}>\frac{n-d+\varkappa}{p_{2}}$. Then for the weight $v_{\varkappa}^{\Gamma}$ introduced in Example 2.13 the embedding

$$
\mathrm{id}: \operatorname{tr}_{\Gamma} B_{p_{1} q_{1}}^{s_{1}}\left(\mathbb{R}^{n}, v_{\varkappa}^{\Gamma}\right) \longrightarrow \operatorname{tr}_{\Gamma} B_{p_{2} q_{2}}^{s_{2}}\left(\mathbb{R}^{n}, v_{\varkappa}^{\Gamma}\right)
$$

is compact and the related entropy numbers satisfy

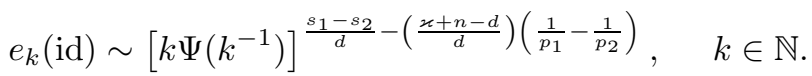

Proof. We follow the proof of Theorem 4.6. We consider Theorem 3.5 and Definition 3.4 and arrive at

$$
\begin{aligned}
e_{k}\left(\mathrm{id}: \operatorname{tr}_{\Gamma} B_{p_{1} q_{1}}^{s_{1}}\left(\mathbb{R}^{n}, v_{\varkappa}^{\Gamma}\right) \longrightarrow\right. & \left.\operatorname{tr}_{\Gamma} B_{p_{2} q_{2}}^{s_{2}}\left(\mathbb{R}^{n}, v_{\varkappa}^{\Gamma}\right)\right) \\
& =e_{k}\left(\mathrm{id}: \mathbb{B}_{p_{1} q_{1}}^{s_{1}-\frac{\varkappa}{p_{1}}-\frac{n-d}{p_{1}}}(\Gamma) \longrightarrow \mathbb{B}_{p_{2} q_{2}}^{s_{2}-\frac{\varkappa}{p_{2}}-\frac{n-d}{p_{2}}}(\Gamma)\right) .
\end{aligned}
$$

Combining this with (4.10) completes the proof.

\section{Approximation numbers of embeddings between weighted Besov spaces}

5.1. Definition and results. In this section we recall the basic definitions and properties concerning approximation numbers and present the results on approximation numbers of the compact trace operator.

Definition 5.1. Let $T \in L(X, Y), k \in \mathbb{N}$. The $k$ th approximation number $a_{k}$ of $T$ is defined by

$$
a_{k}(T)=\inf \{\|T-L\|: L \in L(X, Y), \operatorname{rank} L<k\},
$$

where $\operatorname{rank} L$ is the dimension of the range of $L$.

The approximation numbers have properties analogous to those of the entropy numbers. We present them in the following lemma.

Lemma 5.2. Let $X, Y$ and $Z$ be quasi-Banach spaces, let $S, T \in L(X, Y)$ and $R \in$ $L(Y, Z)$.

(i) (Monotonicity): $\|T\|=a_{1}(T) \geq a_{2}(T) \geq \cdots \geq 0$.

(ii) (Additivity): If $Y$ is a $p$-Banach space $(0<p \leq 1)$, then for all $j, k \in \mathbb{N}$

$$
a_{j+k-1}^{p}(S+T) \leq a_{j}^{p}(S)+a_{k}^{p}(T) .
$$

(iii) (Multiplicativity): For all $j, k \in \mathbb{N}$

$$
a_{j+k-1}(R T) \leq a_{j}(R) a_{k}(T) .
$$

(iv) (Rank property):

$$
a_{n}(T)=0 \text { if, and only if, } \operatorname{rank} T<n .
$$

The best general references here are [CS90] and [ET96]. In the sequel, we restrict ourselves to $d$-sets to formulate our result. Recall that the function $w_{\varkappa}^{\Gamma}$ is a weight introduced in Example 2.4. We now state the main result for approximation numbers.

THEOREM 5.3. Let $0<d<n, 1<p<\infty, \frac{1}{p}+\frac{1}{p^{\prime}}=1, \frac{n-d+\varkappa}{p}<s \leq \frac{n+\varkappa}{p}$, and $-(n-d)<\varkappa$. Let $\Gamma$ be a d-set according to Definition 2.1. Then the trace operator

$$
\operatorname{tr}_{\Gamma}: B_{p p}^{s}\left(\mathbb{R}^{n}, w_{\varkappa}^{\Gamma}\right) \rightarrow L_{p}(\Gamma)
$$


is compact and the related approximation numbers $a_{k}$ satisfy

$$
a_{k}\left(\operatorname{tr}_{\Gamma}: B_{p p}^{s}\left(\mathbb{R}^{n}, w_{\varkappa}^{\Gamma}\right) \rightarrow L_{p}(\Gamma)\right) \sim k^{\frac{1}{d}\left(\frac{n+\varkappa}{p}-s\right)-\frac{1}{p}}, \quad k \in \mathbb{N} .
$$

Proof. As a consequence of Theorem 3.3 and Definition 3.2, from embedding (5.2) we get

$$
\operatorname{tr}_{\Gamma}: B_{p p}^{s}\left(\mathbb{R}^{n}\right) \rightarrow L_{p}(\Gamma) .
$$

Combining this with Theorem 2 and Remark 9 (Example) in [Tri04] we obtain the desired estimate (5.3). The compactness is covered by Proposition 4.8 with $p_{1}=p_{2}=q$.

REMARK 5.4. Note that (4.9) coincides with (5.3) for $p_{1}=p_{2}=q=p$. One should expect a different behaviour of $e_{k}\left(\operatorname{tr}_{\Gamma}\right)$ and $a_{k}\left(\operatorname{tr}_{\Gamma}\right)$ for $p_{1} \neq p_{2}$. This study is postponed to a later occasion, as is the counterpart of Proposition 4.9 for approximation numbers.

Acknowledgments. This is a part of the author's PhD thesis, written under the supervision of Dorothee D. Haroske at the University of Jena. The author wishes to express her deepest appreciation to her for active interest in the preparation of this paper. I also would like to thank Professor Hans Triebel for the helpful discussions and suggestions.

\section{References}

[Bui82] H. Q. Bui, Weighted Besov and Triebel spaces: interpolation by the real method, Hiroshima Math. J. 12 (1982), 581-605.

[Bui84] H. Q. Bui, Characterizations of weighted Besov and Triebel-Lizorkin spaces via temperatures, J. Funct. Anal. 55 (1984), 39-62.

[BPT96] H. Q. Bui, M. Paluszyński and M. H. Taibleson, A maximal function characterization of weighted Besov-Lipschitz and Triebel-Lizorkin spaces, Studia Math. 119 (1996), 219-246.

[BPT97] H. Q. Bui, M. Paluszyński and M. H. Taibleson, Characterization of the BesovLipschitz and Triebel-Lizorkin spaces. The case $q<1$, J. Fourier Anal. Appl., 3 (1997) (Spec. Iss.), 837-846.

[CS90] B. Carl and I. Stephani, Entropy, Compactness and the Approximation of Operators, Cambridge Univ. Press, Cambridge, 1990.

[ET96] D. E. Edmunds and H. Triebel, Function Spaces, Entropy Numbers, Differential Operators, Cambridge Tracts in Mathematics 120, Cambridge University Press, Cambridge, 1996.

[ET98] D. E. Edmunds and H. Triebel, Spectral theory for isotropic fractal drums, C. R. Acad. Sci. Paris 326 (1998), 1269-1274.

[ET99] D. E. Edmunds and H. Triebel, Eigenfrequencies of isotropic fractal drums, Oper. Theory Adv. Appl. 110 (1999), 81-102.

[GR85] J. García-Cuerva and J. L. Rubio de Francia, Weighted Norm Inequalities and Related Topics, North-Holland Mathematics Studies 116, North-Holland, Amsterdam, 1985.

[HP] D. D. Haroske and I. Piotrowska, Atomic decomposition of function spaces with Muckenhoupt weights; an example from fractal geometry, Math. Nachr., to appear.

[HT94a] D. D. Haroske and H. Triebel, Entropy numbers in weighted function spaces and eigenvalue distribution of some degenerate pseudodifferential operators I, Math. Nachr. 167 (1994), 131-156. 
[HT94b] D. D. Haroske and H. Triebel, Entropy numbers in weighted function spaces and eigenvalue distribution of some degenerate pseudodifferential operators II, Math. Nachr. 168 (1994), 109-137.

[KLSS06a] Th. Kühn, H.-G. Leopold, W. Sickel and L. Skrzypczak, Entropy numbers of embeddings of weighted Besov spaces, Constr. Approx. 23 (2006), 61-77.

[KLSS06b] Th. Kühn, H.-G. Leopold, W. Sickel and L. Skrzypczak, Entropy numbers of embeddings of weighted Besov spaces II, J. Differential Equations 15 (2006), 197-211.

[KLSS07] Th. Kühn, H.-G. Leopold, W. Sickel and L. Skrzypczak, Entropy numbers of embeddings of weighted Besov spaces III. Weights of logarithmic type, Math. Z. 255 (2007), 1-15.

[Mou01] S. D. Moura, Function spaces of generalised smoothness, Dissertationes Math. 398 (2001).

[Muc72a] B. Muckenhoupt, Weighted norm inequalities for the Hardy maximal function, Trans. Amer. Math. Soc. 165 (1972), 207-226.

[Pio06] I. Piotrowska, Weighted function spaces and traces on fractals, PhD thesis, Jena, 2006.

[Pio] I. Piotrowska, Traces on fractals of function spaces with Muckenhoupt weights, Functiones et Approximatio, to appear.

[Ste93] E. M. Stein, Harmonic Analysis: Real-Variable Methods, Orthogonality, and Oscillatory Integrals, Princeton Mathematical Series 43, Princeton University Press, Princeton, NJ, 1993.

[ST89] J. O. Strömberg and A. Torchinsky, Weighted Hardy Spaces, LNM 1381, Springer, Berlin, 1989.

[Tri83] H. Triebel, Theory of Function Spaces, Birkhäuser, Basel, 1983.

[Tri92] H. Triebel, Theory of Function Spaces II, Birkhäuser, Basel, 1992.

[Tri97] H. Triebel, Fractals and Spectra, Birkhäuser, Basel, 1997.

[Tri01] H. Triebel, The Structure of Functions, Birkhäuser, Basel, 2001.

[Tri04] H. Triebel, Approximation numbers in function spaces and the distribution of eigenvalues of some fractal elliptic operators, J. Approx. Theory 129 (2004), 1-27.

[Tri06] H. Triebel, Theory of Function Spaces III, Birkhäuser, Basel, 2006. 
